\title{
Reflexiones sobre la instalación de una perspectiva internacional de los Derechos del Niño: un modelo americano de burocratización de la infancia
}

\author{
María Soledad Rojas Novoa*
}

\begin{abstract}
Resumen
En el campo de la "protección de los Derechos del Niño", recurrir a los acuerdos y organismos internacionales resulta fundamental para dar visibilidad a ciertas temáticas y procurar el apoyo político respectivo. El problema surge cuando las categorías que ellos manejan son naturalizadas, restando movilidad al objeto de preocupación y promoviendo nuevas formas de exclusión. Este trabajo busca cuestionar esos espacios de naturalización y develar las negociaciones de las cuales son producto en el continente americano. Una idea central para este trabajo es que estas tensiones han contribuido a la configuración de lo que llamo "un modelo americano de burocratización de la infancia".
\end{abstract}

Palabras clave: Derechos de infancia - organismos internacionales - panamericanismo.

\begin{abstract}
In the field of the studies focused on "Children's Rights protection", the resort to international agreements and organizations becomes essential to give visibility to certain topics and provide pertinent political support. The problem lies in the naturalization of the standards employed, reducing mobility to subjects concerned and promoting new forms of exclusion. This study seeks to question this naturalized fields and disclose the "negotiations" responsible at this juncture in the American continent. The main idea for this study is that these tensions have contributed to the shaping of what I name "the American model of childhood bureaucratization".
\end{abstract}

Keywords: Children's rights - international organisms - panamericanism.

* Psicóloga, Universidad Diego Portales. Master en Psychanalyse et Lien Social, Universidad París VII. Candidata a Doctora en Sociología, Centre de Sociologie des Pratiques et des Représentations Politiques, Universidad París VII (becaria de CONICYT). Correo electrónico: soledad.rojasnovoa@gmail.com 


\section{INTRODUCCIÓN: MIRADAS A UN MODELO INTERNACIONAL DE LOS DERECHOS DEL NIÑO}

La situación mundial de la infancia hacia fines de 1980 estuvo marcada por una serie de condiciones consideradas como criticables por la comunidad internacional: tasas de mortalidad elevadas, acceso deficiente a la salud y a la educación, niños maltratados, explotados, encarcelados, niños refugiados y víctimas de conflictos armados, entre otras. Inspirados por la intención de una regulación homologable entre ellos, los Estados han solicitado la elaboración de un texto donde los derechos del niño fuesen anunciados en detalle y tuviesen fuerza de ley desde el derecho internacional. Frente a esta petición, en 1989 la ONU ha aprobado unánimemente el texto de la Convención de los Derechos del Niño (CDN). Este documento fue ratificado por veinte Estados en 1990 y deviene el Tratado de los Derechos del Hombre que ha sido más larga y rápidamente ratificado; a la fecha, sólo dos países no lo han hecho: Estados Unidos y Somalia.

La firma de la CDN es un hito para los avances en materia de Derechos Humanos, sin embargo es sólo un momento dentro de un proceso mayor de movilización respecto de los derechos de infancia. En efecto, la doctrina de los derechos del niño tiene un largo recorrido en Europa occidental y Estados Unidos, así como también en Latinoamérica y, lejos de ser una propuesta que nace con la firma de la CDN, sus raíces pueden rastrearse bastante antes.

Como parte de un fenómeno de índole mundial, este movimiento se origina a finales del siglo XIX, particularmente en los movimientos de reforma legal que argumentaban como propósito proteger a los niños de la explotación laboral y generar mecanismos de rehabilitación para los infractores. Existía además una preocupación desde ámbitos religiosos y un contingente de médicos que -acorde al movimiento sanitario de la época- se encontraban preocupados por lograr un descenso en las tasas de mortalidad infantil.

Si bien la "sensibilidad" en relación con los niños se fue fortaleciendo durante la segunda mitad del siglo XIX, es a comienzos del siglo XX que podría considerarse como asentada la propuesta, por lo menos a nivel institucional, de asegurar para los niños algún nivel de bienestar y protección. Como decíamos, la influencia cultural europea y luego norteamericana fue clave para la difusión de este enfoque (Rojas, 2007).

El movimiento de esos años se registra en una serie de reuniones científicas realizadas principalmente en París, Bruselas y Washington, y en la fundación de diferentes instituciones dedicadas a la promoción del bienestar de los niños, entre ellas la asociación Save the Children (Londres, 1919), I'Union Internationale de Secours aux Enfants (Ginebra, 1920) o la Association Internationale pour la Protection de l'Enfance (Bruselas, 1921).

En particular para el continente americano, en 1912 se fundaba el Children's Bureau en Estados Unidos. En 1913, Argentina organizaba el Primer Congreso Nacional del Niño, actividad que fue proliferando en diferentes capitales de las Américas, generando además códigos nacionales de respeto al bienestar de la infancia. A nivel regional, en 1916 se realiza el Primer Congreso Panamericano del Niño, reunión que perdura hasta nuestros días. En 
un intento por unificar estas actividades que permanecían aún discontinuas, se funda en Montevideo, en 1927, el Instituto Internacional Americano de Protección a la Infancia ${ }^{1}$.

En materia de tratados, podríamos señalar tres hitos relevantes:

1. En 1923, la Fundación Save the Children redactó una Declaración de los Derechos del Niño, documento que fue ratificado por la Asamblea de la Sociedad de Naciones en 1924, dando paso a la Ilamada "Declaración de Ginebra". Este documento fue la primera sistematización de unos derechos de infancia y, si bien ha sido concebido como un Ilamado solidario, sin ninguna autoridad legal, es considerado un texto inaugurador para la movilización de acuerdos en materia de derechos de infancia.

2. En 1959, la Asamblea General de Naciones Unidas aprueba de forma unánime una nueva Declaración de los Derechos del Niño. Esta Declaración, además de los principios genéricos ya conocidos ("derecho del niño a ser niño", "derecho del niño a ser feliz"), introduce una gama de derechos (salud, alimentación, educación) a ser atendidos por los Estados y, si bien continúa en el registro de un documento no constriñente, les recomienda explícitamente -junto a los organismos especializados y a las ONGs- dar máxima atención y promoción a los artículos que ella propone.

3. Luego, en 1989, la Convención sobre los Derechos del Niño se convierte en el primer instrumento internacional jurídicamente vinculante que, mediante la ratificación o la adhesión, los gobiernos nacionales se comprometen a respetar. La CDN se sitúa así como un punto cúlmine para el reconocimiento de los derechos de la infancia y el anhelado arribo a un consenso internacional para que los Estados sean responsables de ese reconocimiento.

Así las cosas, la aparente fluidez de este recorrido suele sugerir que, una vez logrado este anhelo y ya establecidas las bases de las declaraciones y convenciones en la materia, el único trabajo que quedaría por delante sería hacerlas cumplir. Dicho de otra manera, que luego de un largo período de investigación y negociaciones, finalmente podríamos descansar en la conclusión de que las intervenciones en derechos de infancia tienen un fin último llamado "el interés superior del niño", y que el trabajo conjunto de los Estados y los organismos internacionales nos dirá cuál es ese interés y cómo trabajar con él.

Tal como plantea Vanessa Pupavac (2001: 96), "quizás por la virtualidad de la ratificación de la CDN, sus beneficios son tratados como axiomáticos y su instalación representa una progresión natural de los esfuerzos que le anteceden. Esta fijación de la CDN como momento cúlmine, no considera las 'olas' de significado para el derecho ni el significado del derecho en sí mismo; la metáfora de 'olas' apunta a las contradicciones inherentes a las distintas generaciones de derecho".

1 Hoy Instituto Interamericano del Niño, la Niña y Adolescentes (IIN). 
Lo que esta metáfora devela es que todos los pasos dados para ir otorgando solidez al engranaje simbólico que configura la categoría de los derechos de infancia contenida en la CDN proveen, al mismo tiempo, del más alto tambaleo. Esto porque, si bien las declaraciones internacionales podrían proponerse como el resultado de un consenso, ellas deben también pensarse como fruto de las negociaciones entre diferentes fuerzas y filosofías, determinadas por un contexto histórico, cultural, económico y social particular, y sólo el cuestionamiento de esas bases permitirá dar movilidad a las categorías construidas.

En este punto, la historiadora Paula Fass (2001) plantea que el recorrido hasta el establecimiento de la CDN ha sido impredecible y sinuoso, y que este documento será posible de comprender sólo teniendo en consideración la recreación de los roles de género, la preponderancia de las disciplinas concernidas y los asuntos relativos al poder compatibles con la estética del siglo XX.

Este artículo considera que, para el caso del continente americano, aquellas variables pueden representarse en tres características singulares: primero, la influencia de los movimientos feministas; segundo, la introducción de las técnicas sociales en el trabajo con las familias; y tercero, la representatividad adquirida por los organismos internacionales. Una vez esbozados estos ejes podemos dilucidar las tensiones que ha comprendido la instalación de la CDN en la región y la particularidad que ella toma en los intentos nacionales por generar medidas propias, los esfuerzos regionales por unificar estas experiencias y la influencia de modelos y prácticas extracontinentales.

El desarrollo que se presenta a continuación avanza en gran medida sobre la articulación de los enclaves históricos, políticos y económicos que sostienen la gestión de dos instancias regionales preponderantes y de trabajo conjunto en el campo de los derechos de infancia en la región: el Congreso Panamericano del Niño y el Instituto Interamericano del Niño, la Niña y Adolescentes. La representatividad que estos organismos van alcanzando a nivel internacional a lo largo del siglo XX y las alianzas estratégicas que ellos condicionan para el movimiento de protección de la infancia en la región aportan en gran medida a nuestro intento por cuestionar ese recorrido "impredecible y sinuoso" de la CDN en América.

Teniendo estos elementos a disposición, al final de este trabajo ofrecemos algunas reflexiones que intentan problematizar aquello que llamamos "un modelo americano de burocratización de la infancia".

\section{RECORRIDO PARA UNA INSTALACIÓN REGIONAL DE LA CONVENCIÓN DE LOS DERECHOS DEL NIÑO}

\subsection{Relevancia de los movimientos feministas en el esfuerzo americano de protección de la infancia}

La protección de las condiciones de vida de niños, niñas y adolescentes de la región viene problematizándose desde principios del siglo XX y su aparición como asunto de 
preocupación social -los inicios del Ilamado "movimiento americano de la protección de la infancia" - se relaciona directamente con la expansión de los derechos de la mujer.

En efecto, el interés de grupos feministas por promover los asuntos de la relación madrehijo encuentra sus raíces en el Primer Congreso Nacional del Niño, celebrado en Argentina en 1913. Con este evento las feministas argentinas inauguran oficialmente su lucha por vincular los derechos de las mujeres a los de la infancia, teniendo como eje central una demanda al Estado de sostener las instituciones que deberían proteger a los niños y a las madres de clase trabajadora.

En 1916, un grupo ampliado de este movimiento organiza en Buenos Aires el Primer Congreso Panamericano del Niño, contexto en el que su demanda encuentra los primeros espacios de conflicto con posturas discrepantes, especialmente con aquellas provenientes de la medicina (liderada por hombres, en su mayoría latinoamericanos). El desencuentro entre estos grupos tiene que ver sobre todo con la valorización que se hace del binomio madrehijo: en la lógica médica, y considerando el particular interés de la época por reducir las tasas de mortalidad infantil, la madre aparece como una "variable higiénico-profiláctica", ligada a un modelo de prevención que promueve el rol de la madre cuidadora (y el padre proveedor), encargada y responsable de la salud de los niños. En este sentido, el interés de los médicos por intervenir en las familias permanece en una lógica muy lejana al énfasis propuesto por las representantes de un "feminismo maternalista" que buscaba más bien un cambio social en relación con la posición de la mujer.

Cuando el grupo de feministas latinoamericanas organizaba el Segundo Congreso Panamericano en Montevideo en 1919, ya había perdido gran representatividad; si bien una mujer, la Dra. Paulina Luisi, presidía el Congreso, los médicos, abogados y educadores ya habían comenzado a afianzar el liderazgo en este contexto. Los dos congresos siguientes, Río de Janeiro en 1922 y Santiago en 1924, ofrecieron cada vez menos espacio a las feministas latinoamericanas y sirvieron de escenario para el establecimiento de las bases de un nuevo proyecto regional en el cual ellas definitivamente no tenían cabida.

Así las cosas, con la exclusión estratégica de estas mujeres de avanzada, se fue concretando la idea de crear una Oficina Internacional Americana de Protección a la Infancia, que más tarde tomaría forma en la propuesta de un Instituto Internacional Americano de Protección a la Infancia (IIAPI). El 9 de junio de 1927, diez países de las Américas ${ }^{2}$ suscriben el acta de fundación del IIAPI, quedando definitivamente constituido, con sede central en Montevideo y bajo la administración del Dr. Luis Morquio, el Dr. Roberto Berro y el Dr. Víctor Escardó y Anaya.

En un análisis de esta iniciativa, la investigadora estadounidense Donna Guy (1998a: 456) concluye que las bases de este organismo soslayaban el rol específico de las madres,

2 Esos diez países son Argentina, Bolivia, Brasil, Cuba, Chile, Ecuador, Estados Unidos, Perú, Uruguay y Venezuela. Con el correr de los años paulatinamente se irán sumando otros países, hasta que en 1949 habrán adherido todos los gobiernos de las Américas y el Caribe. 
situando a la familia como una unidad basada en el monitoreo de los niños pobres por los médicos de clase media, procurando "crear la familia de clase obrera moderna que supliría la futura generación de ciudadanos".

Acorde con este análisis entran en valoración las ideas promulgadas por las feministas estadounidenses representantes del Children's Bureau, agencia nacional de los Estados Unidos a cargo de la protección de la infancia y la familia. A diferencia de las feministas latinoamericanas, este poderoso grupo de élite estadounidense buscaba promover una cooperación entre profesionales hombres y mujeres, sosteniendo un comprometido apoyo al IIAPI tanto en lo que refiere a sus prioridades programáticas como a sus necesidades financieras. La novedad del aporte de estas mujeres es considerar que los problemas que aquejan a la infancia podrían ser disminuidos mediante medidas preventivas, siendo el Trabajo Social la vía por excelencia: "si las naciones querían disminuir la delincuencia juvenil debían ayudar a los padres a monitorear a sus hijos y no cargar al Estado de la necesidad de especialistas en delincuencia" (Guy, 1998a: 458).

Esta distancia entre la postura de las feministas latinoamericanas y las del Children's Bureau da cuenta del nivel en que el género justificó la intervención en las familias con propósitos muy distintos. A grandes rasgos, mientras el primer grupo demandaba al Estado las condiciones básicas para sustentar el rol de la mujer como madre/trabajadora, el segundo grupo promovía el rol de la caridad elitista privada, cambiando la perspectiva feminista de demanda al Estado hacia una estrategia flexible con foco en el niño y la familia.

Desde la perspectiva de Donna Guy (1998, 1998a), introducir la propuesta del Children's Bureau resultaba ideal para los planes de una Reforma Infantil Panamericana y era particularmente Ilamativa para los líderes del IIAPI ya que sería, en última instancia, más económica, al tiempo que ayudaba a diluir las tensiones que se instalaban entre las autoridades de Estado y la caridad privada.

Para 1928, el Dr. Luis Morquio pronunciaba un discurso en el Congreso Internacional de Protección de la Infancia en París, que por primera vez sitúa al Servicio Social dentro de las disciplinas de mayor relevancia para el trabajo con las familias en América: "Un elemento nuevo se ha incorporado a los servicios de asistencia y de defensa social que tiene hoy un papel preponderante y que ha sido la nota culminante de esta reunión de Congresos: el Servicio Social, es decir, una institución independiente, autónoma, que tiene por objeto analizar cada caso individualmente, aportando o indicando los medios preventivos o efectivos, convenientes o necesarios, para prevenir el mal o para curarlo" (IIAPI, 1929: 260).

\subsection{La introducción de las técnicas sociales y la regulación de las familias}

La pregunta por los problemas sociales implica una indagación de los procesos mediante los cuales ciertas temáticas adquieren relevancia y visibilidad, movilizando fondos o frentes de acción específicos. Los modos en que las leyes son traducidas en la práctica (y sus consecuencias particulares) dependen de relaciones de poder forjadas en contextos históricos determinados y expresados en categorías semánticas precisas. Por ello, en distintas 
circunstancias, ciertas categorías serán elegidas como merecedoras de campañas en defensa de los derechos humanos y otras quedarán a la espera, algunas técnicas y enfoques serán prioritarios, otros serán desechados. Finalmente lo que consta como más urgente no dependerá tanto de la realidad como de las prioridades mediáticas y de la gestión política.

Para comprender la creación de esta categoría de "malos elementos sociales" en relación a la infancia, digamos "los niños de la calle" o "los niños trabajadores", Sandra Carreras y Bárbara Potthast (2005) tienen la hipótesis de que ellos se sitúan como "amenazantes" e instalan la preocupación por regularlos en tanto denuncian una crisis económica y familiar. Siguiendo la lógica de estas investigadoras, aquel interés que médicos, higienistas y abogados, representantes de la gestión panamericana, tienen por una "infancia en peligro", se genera realmente menos por los niños y sus familias que por la consolidación del Estado y el poder de los grupos interesados. Estos niños, sobre los que habría que prevenir o curar, representan, frente a los higienistas y a las autoridades en general, la manifestación de una destrucción del valor de la familia y, con ello, su simple existencia significaría una amenaza al orden social, al menos simbólicamente, en tanto ponen en peligro el ideal de lo que ellos representan: "el futuro y el progreso de las naciones americanas".

En palabras del Dr. Roberto Berro, Director del IIAPI entre 1935 y 1956 :

“Es una necesidad mayor cuidar al niño, al que ha nacido y que está en germen; al que es la alegría de un hogar y al que tiene la desgracia inmensa, quizás irreparable, de haberlo perdido; al que tiene a mano todas las oportunidades que reclaman para él los pensamientos de los paidólogos y los votos de los Congresos y de aquellos que no gozan de estas oportunidades pero que el Estado moderno y consciente quiere y debe proveérselas; a aquellos de constitución normal y que se desarrollan bajo el contralor y la orientación de un hogar sano y correcto, y a aquellos desgraciados o descuidados, que por vicios y defectos constitucionales y ambientales están en peligro de apartarse, o se apartaron ya, de las normas éticas de la corrección y de la convivencia social. A todos los niños en fin, porque es con la suma de todos ellos que ha de formar cada pueblo su propio porvenir; que no está basado -ni siquiera en los países de grandes riquezas naturales- nada más que en la cultura física, moral e intelectual de la población, porque el HOMBRE es el real valor, la riqueza positiva de una nación. Mayor necesidad de cuidar al niño como tal, pero hacerlo recordando siempre que su completo bienestar radica en la integralidad de un concepto social inapartable: la constitución de una familia. Aquí está, señoras y señores, la esencia misma del problema. Los hechos repetidos de la vida real, y los razonamientos diversificados de los altos pensadores, comprueban bien la afirmación, que no debe ser vulgar a pesar de lo repetida, de que la Familia es la célula base del conglomerado social. Y esta célula no puede ser desintegrada; sería el fin de la sociedad" (IIN, 1956: 388-389)32.

Estas afirmaciones son altamente representativas del espíritu de la gestión del IIAPI en esos años y bastante esclarecedoras de la lógica que va a imponerse: la introducción de las técnicas de lo social permite situar a la familia como un elemento discontinuo de la

3 Discurso pronunciado por Roberto Berro en la Universidad de Chile en 1945, citado por Elisabeth Enochs en 1956 durante un homenaje conmemorativo de la muerte de Berro. 
sociedad propicio para una serie de intervenciones correctivas aisladas y eficaces. La familia sería, finalmente, un laboratorio de observación de conductas populares y una plataforma de lanzamiento de tácticas que contrarían los efectos sociales "negativos" o "no deseados".

Así las cosas, las propuestas para regular la infancia aparecen, en palabras de Jacques Donzelot (1977), como "la encarnación de la figura de misionero civilizador del cuerpo social", en tanto van obligando a las familias a un comportamiento que implica "vigilar" ellas mismas a sus hijos, contando con la "compañía" y "apoyo" de esos representantes de "lo social".

Ya sea un mecanismo de reemplazo del poder jurídico por el saber de las ciencias sociales -que la prevención reprima "en nombre del saber"- o sea una extensión misma de lo jurídico -refinamiento de sus procedimientos y ramificación del poder-, estas nuevas "técnicas de lo social" se caracterizan por movilizar un mínimo de coerción para obtener un máximo de intromisión en las familias y lograr reorganizar los intercambios entre adultos y niños en función de imperativos socioeconómicos.

Ante este escenario, es plausible la hipótesis según la cual la doctrina de los derechos del niño viene a instalarse en el lugar vacante que ha dejado disponible una familia que falla a los ideales establecidos, ya que, tal como nos dice Valeria Llobet (2010), en condiciones donde la familia pudiese cumplir ese ideal y contener a sus hijos no sería necesaria una institución para defenderlos.

El Código de Menores menciona: "Corresponde al Estado ejercer por medio de las instituciones adecuadas, la vigilancia y protección del menor desde su concepción hasta la edad de diez y ocho años, en cuanto no sean ejercidas por los padres o demás personas a quienes la ley obliga a ese deber o confiere tal atribución (...), y deberá estimular y proveer al bienestar de la familia como el método más indicado para la seguridad material y moral del menor" (IIAPI, 1948: 47).

En esta misma línea, los historiadores Pablo Rodríguez y María Emma Manarelli (2007) proponen que el "problema social" que se va construyendo en relación a la infancia se instala en el espacio abierto por el intercambio de autoridad entre los lazos de parentalidad y las autoridades públicas. Una de las lecturas para ese intercambio es que introduce toda una conceptualización de la familia como pieza central del dispositivo de moralización y de control de las clases populares, en tanto la adopción de una conducta familiar racionalizada, definida sobre la base de observaciones discontinuas que aíslan un "modelo sano", "protegido" o "en situación regular", tiene que ver con fijar a los individuos, regulando simultáneamente las relaciones familiares y sociales. En este orden de cosas, Ilegamos a la conclusión de que la asociación de los niños con el espacio privado es un discurso prescriptivo y no descriptivo.

Tal como diría recientemente el abogado uruguayo Didier Opertti (2009) en el XX Congreso Panamericano del Niño (Lima, 2009), "que se acabe aquella vieja historia de que el hogar era un recinto sagrado al que no podía penetrar el Estado, la figura de un Estado que quiere regular conductas que cuando llegan al terreno patológico, requieren de regulación". 


\subsection{Instancias internacionales y la ampliación del modelo}

Empezando por Brasil (1927), Costa Rica (1932) y Uruguay (1934), se observa un alza en la producción de reformas que tienden a la regulación de esa infancia clasificada como "abandonada" o "delincuente". A esta clasificación responde una serie de Códigos Nacionales del Niño que, según la opinión de Donna Guy, y en particular para el contexto latinoamericano, "explícitamente definen las relaciones familiares y limitan los derechos de custodia de las mujeres casadas con sus hijos, presentan vacíos en las leyes de adopción y carecen de un lenguaje claro que resguarde los derechos y responsabilidades de las madres" (Guy, 1998a: 453).

Ya para 1948 se promulga el Código Panamericano del Niño. A grandes rasgos, este Código no sólo reconoció que todos los niños y niñas son sujeto de derechos, sino que también estableció la potestad y el deber del Estado de proteger a los niños, al tiempo que otorgó la responsabilidad directa sobre su bienestar a la familia. Como bien sugiere Donna Guy (1998), este Código podría ser pensado como un antes y un después en el movimiento de los derechos del niño en América: como veníamos anticipando, instala oficialmente una transición del foco de interés en el Estado hacia el niño y la familia, admitiendo un cambio de demandas que, si bien continúan interpelando al Estado, ya no lo hacen de modo exclusivo.

Al año siguiente de la promulgación del Código Panamericano del Niño en 1949, la Unión Panamericana se transforma en la Organización de Estados Americanos (OEA), instalando un nuevo Programa de Cooperación Técnica para enfrentar los problemas del desarrollo económico y social de la región, entre ellos los asuntos que tienen que ver con la infancia y la adolescencia.

En este proceso, el IIAPI es invitado a incorporarse a la OEA como uno de sus organismos especializados ${ }^{4}$. Ampliando su objetivo histórico de ser un centro de recopilación de información, se le asigna la función específica de promover el estudio de problemáticas relativas a la infancia en las Américas -definir prioridades y objetivos de trabajo- y generar instrumentos técnicos para abordarlos.

En palabras del profesor Roberto Berro, director del Instituto por esos años, "lo que cabe consignar aquí es la importancia del hecho que, si bien mucho nos honra, nos da también una responsabilidad, pues nos obliga a trabajar con intensidad creciente para merecer la confianza que se ha puesto en el Organismo que, muy modesto en sus comienzos, ha ido -lenta pero progresivamente- manteniendo y elevando su jerarquía moral e intelectual" (IIAPI, 1949: 2).

4 No hay que olvidar que el IIN es el único Organismo Especializado de la OEA que fue anexado, es decir, que existía independientemente y que no fue fundado en relación con la OEA. Se habían creado con anterioridad: la Organización Panamericana de la Salud (1902), la Comisión Interamericana de Mujeres (1928), el Instituto Panamericano de Geografía e Historia (1928), el Instituto Indigenista Interamericano (1940), el Instituto Interamericano de Cooperación para la Agricultura (1942). 
En efecto, la anexión a la OEA implica para el Instituto situarse en una posición clave para el continente y, con ello, resiente un fuerte período de asimilación y reestructuración, tanto en su relación con las instancias internacionales como al interior del mismo Organismo.

Casi diez años después de la anexión, durante la XXXVIII Reunión del Consejo Directivo del Instituto (Lima, 1957) se coronan estas transformaciones, primero con su cambio de nombre, pasando a llamarse desde ahora "Instituto Interamericano del Niño" (IIN) $)^{5}$, y luego con la integración del Congreso Panamericano del Niño a su gestión de manera que, conservando el lugar que ha alcanzado como conferencia especializada de la OEA, el Congreso desde ahora pasa a ser organizado y difundido bajo responsabilidad del Instituto.

El período entre 1961 y 1963 avanza un paso más en este proceso, tomándose la decisión de anexar al IIN a la Secretaría General de la OEA, por lo que ahora contará con un doble estatuto dentro de esta Organización. Lo que esta doble condición implica es que el IIN adhiere al presupuesto de la OEA, a condición de dejar a decisión de ella todo lo que respecta a su Programa: desde ahora el IIN dispondrá sólo de autonomía técnica.

Dadas estas condiciones, el período 1962-1963 se califica como uno de los más difíciles desde la fundación del IIN. Queda consignado que en los años preparatorios para la presentación de este nuevo acuerdo hubo grandes discrepancias entre los miembros del Consejo Directivo ya que algunos eran reticentes a ceder sus facultades como producto de coordinar acciones entre organismos con fines e historias tan diversas (Acta XL, 1959). Sin duda queda fuera de este trabajo un análisis de los fundamentos y el modelo de gestión de la OEA. Lo que más bien nos interesa destacar es que evidentemente alcanzar este nivel de representatividad a nivel regional tendría para el IIN el precio de una enorme reestructuración y de contundentes concesiones que lo dejarán, en última instancia, reducido a un organismo técnico, cuya mayor incidencia es el asesoramiento de los Estados.

El gran ajuste que el IIN debe hacer en razón de su nuevo posicionamiento regional tiene consecuencias inmediatas y visibles para el alcance internacional del movimiento americano de protección de la infancia y para los modos en que se establecen relaciones con los organismos que comparten este campo de acción. Entre ellos podemos contar el Fondo de las Naciones Unidas para la Infancia (UNICEF), fundado en 1946; el Centre International de l'Enfance (CIE), fundado en 1947 por petición del gobierno francés a UNICEF; y el Children's Bureau, que había sido fundado en Estados Unidos en 1912 como división del Departamento de Trabajo de ese país, y que en 1946 es transferido al Departamento de Seguridad Social, cambiando su foco de atención, antes puesto en la regulación de la legislación laboral infantil, a los asuntos de protección social que primaban como preocupación por esos años.

5 Con este cambio de nombre se adapta a la denominación común de los Organismos Especializados de la OEA. Luego, será durante la 79ª Reunión del Consejo Directivo (México, 2004) que, por petición de la representante de Nicaragua y luego de un largo debate, se introducen las diferencias de edad y la perspectiva de género quedando el Instituto designado como 'Instituto Interamericano del Niño, la Niña y Adolescentes'. 
El IIN será, de ahora en más, el referente técnico de la región interamericana, contando con participación discreta en los Estados y con el Congreso Panamericano como instancia privilegiada para un intercambio de información a nivel internacional.

\section{LA CONVENCIÓN DE LOS DERECHOS DEL NIÑO}

El recorrido que venimos de describir se abre paso en un álgido período de negociaciones en materia de protección de la infancia, cuyo punto de referencia fue la Declaración de los Derechos del Niño de 1924 y luego la de 1959. Como veníamos anticipando, ese debate y en general toda la reestructuración a nivel internacional que vivieron las instituciones relativas a la protección de la infancia se caracteriza en gran medida por el interés de fomentar la cooperación multinacional en estos asuntos.

En esta línea, el año 1979 es declarado por la ONU como el Año Internacional de los Derechos del Niño, con el objetivo último de dispersar una idea central: “(...) que los niños particularmente los más vulnerables, son responsabilidad colectiva de la comunidad internacional y que las medidas en beneficio de los niños deben adoptarse en un esfuerzo colectivo tanto de los países desarrollados, como de los países en desarrollo. Concretamente, se quiere estimular la participación de los Ministerios gubernamentales más importantes, los encargados de adoptar decisiones, las Comisiones Nacionales, los Representantes del Sistema de las Naciones Unidas y de la Organización de los Estados Americanos, las organizaciones no gubernamentales y los medios de información" (IIN, 1979: 37).

El retorno al énfasis en la "cooperación horizontal" de este período se justifica, entre otras razones, en el auge de las "áreas de especialización" en el campo de la infancia, en las limitaciones financieras internacionales (entre ellas, la crisis de la OEA en la década de 1980) y en la magnitud de los desafíos que se plantean cuando se busca "ayudar a cada niño de la región". Estas cuestiones profundizan el imperativo de fomentar el trabajo en conjunto y de impedir la duplicación de funciones. Bajo este criterio, el IIN se ve obligado a ampliar su red de contactos y relaciones con el fin de multiplicar recursos en algunos casos y, en otros, de servir como entidad ejecutora de planes y proyectos.

A largo plazo, se pretende gestar un verdadero programa de "cooperación horizontal" (promovido por la OEA y la ONU) que considera la asistencia y capacitación de los Estados por parte de los organismos internacionales, con el fin de que logren una gestión autónoma que permita al mismo tiempo la participación del sector privado y las ONGs.

El IIN, como organismo internacional y en consonancia con la adhesión progresiva de los Estados americanos, se hace parte de este movimiento que en 1989 se corona con la firma de la Convención Internacional de los Derechos del Niño. Por esos años declara: "Es nuestra esperanza que los esfuerzos culminados con la aprobación de la Convención sean complementados por una adecuada promoción del texto y el espíritu de la misma, que se concrete posteriormente en políticas y acciones de los gobiernos y de la comunidad americana. Es en esa dirección que apunta el Plan de Acción Interamericano de la Niñez Carenciada que tiene a su cargo actualmente el IIN" (IIN, 1990: 5). 
Como vemos, la CDN se sitúa como un "punto cúlmine" del cual se desprende linealmente un plan de acción a cumplir. En particular para los países de América Latina y el Caribe, esa conceptualización de la "niñez carenciada" debiese apuntar al modo en que la distribución de los ingresos y los niveles agudos de pobreza conforman un marco que influye directamente en la situación de la infancia y la familia, sobre todo porque el desarrollo económico, social y cultural, así como los acontecimientos políticos, incidieron directamente en la configuración de un marco en el cual América configuró una extensa legislación para la minoridad en situación irregular o en conflicto social, los niños abandonados, los niños de la calle y el trabajo infantil (IIN, 1990).

Específicamente para el IIN, la tarea en este contexto queda plasmada en unos objetivos oficiales que apuntarían a promover regionalmente el "cumplimiento" de la CDN, contribuir al desarrollo de políticas sociales de infancia, articular la relación del Estado con la sociedad civil, promover la cooperación horizontal entre Estados y potenciar acciones en países de menor desarrollo relativo. Asimismo debería procurar un entendimiento con el Comité de los Derechos del Niño para prestar asistencia a los Estados Miembros de la OEA en la elaboración de los informes periódicos al Comité (IIN, 1990).

Esta lógica intenta posicionar para la opinión pública la figura de la CDN como "la demostración misma" de que el campo de protección de la niñez es una variable más en la senda del fortalecimiento de la democracia, la modernización del Estado y el respeto a los derechos humanos, sin evidenciar un hecho central y es que los países latinoamericanos, en pleno período de democratización, incorporaron la CDN en el marco de las reformas de Estado y del "predominio neoliberal" en su concepción y organización del bienestar, la economía y la política, cuestión esencial en términos de las consecuencias en los modos de concebir el Estado y el lugar de la sociedad civil, en las posibilidades de conceptualizar la política social y en las maneras de definir el rol de las instancias internacionales.

Para Donna Guy (1998) esto tiene raíces anteriores ya que, según su perspectiva, ya desde 1960 los cambios en materia de protección infantil en el continente americano debían haber sido atribuidos más a la Alianza para el Progreso que al IIN pues, de la mano del Congreso Panamericano del Niño, paulatinamente fueron perdiendo representatividad. La hipótesis de Guy es que desde esos años el panamericanismo estaba mucho más preocupado por asuntos militares que sociales y que lo que queda de preocupación por los problemas sociales se juega en términos de su moralización, sobre todo en cuestiones de desarrollo. Como sabemos, dicha moralización impacta deslegitimando a los países "incapaces" de cumplir con las normas establecidas internacionalmente, siendo las sociedades débiles y pobres del sur culpables de sus condiciones; las familias en esas sociedades estarán destinadas a ser objeto de intervenciones correctivas para ajustarse a lo establecido, al mismo tiempo que serán interpeladas a una gestión constante para revertir este orden en apariencia natural que determina que algunos "puedan ajustarse" y otros no.

En este sentido, otro elemento central que normalmente no se tiene en cuenta es que todo el debate que sostiene la definitiva instalación de la CDN se enmarca en la Guerra Fría y se caracteriza por la lucha de intereses entre dos grandes bloques: uno encabezado por 
Polonia, que propuso el primer borrador para la CDN, privilegiando los derechos económicos, sociales y culturales como base de toda concepción igualitarista de los derechos humanos, y otro, encabezado por Inglaterra, Francia y Estados Unidos, que concebía el derecho a la libertad -traducido como derechos civiles y políticos- como el derecho a partir del cual deberían derivarse los demás. En esa pugna, la influencia occidental terminó aportando a una modernización entendida justamente como "occidentalización", intentando traer a los países en desarrollo hacia ese bloque. Incluso con el fin de la Guerra Fría persistirá la política occidental de donaciones en la política internacional. El sur es reconceptualizado como "zona de riesgo", modificando el comportamiento cultural. Así las cosas, "la infancia del sur no sólo es borrada de la visión internacional sino que la infancia occidental deviene el modelo para juzgar a las sociedades del sur" (Pupavac, 2001: 101).

En efecto, la gestión de los organismos internacionales en este contexto implica un desafío a la soberanía de los Estados, haciendo un cuestionamiento a los modos de vida de sus ciudadanos. No es casual que Estados Unidos sea uno de los dos únicos países en el mundo que no han ratificado la Convención (el otro, recordemos, es Somalia), aunque tiene alta incidencia en las decisiones tomadas en el Consejo del IIN (sobre todo dada su incidencia en la OEA):

"La condena moral del Sur da sentido a la misión de Occidente: el trabajo en infancia en el Sur se caracteriza por repetir el colonialismo paternalista donde el Norte-Adulto ofrece ayuda y conocimiento al Sur-Niño. En términos del modelo universal de los derechos del niño, la CDN significa la desaparición del derecho de soberanía y de autodeterminación de las sociedades no-occidentales y la legalización de la inequidad internacional, bajo la apariencia de una nueva moral universal" (Pupavac, 2001: 101).

\section{CONCLUSIONES: UN MODELO AMERICANO DE BUROCRATIZACIÓN DE LA INFANCIA}

En el recorrido que hemos intentado esbozar (y aunque mucho quede por indagar en próximas reflexiones) algunas luces encontramos sobre el modo en que ciertas categorías han venido configurando un campo de intervención sobre la infancia en América y los mecanismos mediante los cuales ellas se sitúan como relevantes en congruencia con las relaciones de poder a las cuales están sujetas.

Este campo de preocupación se sostiene sobre la premisa de que la "integralidad" de la familia es "la esencia misma" del problema que sufre un grupo de niños y niñas catalogados como "infancia en peligro" o "infancia peligrosa", de modo que una categoría de "derechos de la infancia" aparecerían ahí donde la familia ha fallado en su función.

Avanzando sobre esta hipótesis, es posible pensar que más que intentar subsanar la falla misma, algo así como una "Convención de los Derechos del Niño" se presta para perpetuar o naturalizar lo que serían las causas de esa "falla":

“La CDN pudo ser concebida debido a que 'algo' vagamente definible como un proceso de democratización de las relaciones sociales -sobre todo al interior de la familia- estaba (y está) en proceso de desarrollo. Pero si movimientos en el campo de lo social hicieron 
pensable la CDN, fue porque el carácter incompleto de éstos la volvieron necesaria. En otras palabras, la igualdad y no discriminación que postulan los instrumentos de derechos humanos resultan -paradójica pero invariablemente- de su inexistencia en la realidad. Si todos los hombres fueran real y naturalmente iguales, los postulados de los derechos humanos serían superfluos. La igualdad humana no existe en la naturaleza; necesita siempre un hecho político fundante" (García Méndez, en Bustelo, 2011: 12).

Ese hecho fundante será difícil de encontrar en un sistema que debilita la participación de sus miembros en el proyecto de bien común y que reprime los propósitos colectivos al que cada miembro puede y debe contribuir. Finalmente, el proyecto colectivo desaparece y en su lugar emerge una serie de desafíos privados con un resultado claro: mientras los logros de algunos signifiquen inevitablemente el fracaso de otros, el sistema se esforzará por la naturalización de las diferencias que produce.

La instalación y expansión de esta lógica en el modelo americano tiene como consecuencia la institucionalización y la universalización de un régimen de protección de la infancia que enfatiza causas individuales y la necesidad de intervenciones profesionales discontinuas, restando importancia al conjunto de circunstancias sociales, políticas y económicas implicadas. En un despliegue progresivo hacia formas individualizadas de la experiencia social, el blanco de las políticas públicas serán directamente los niños, las niñas y sus familias pero no las dinámicas sociales que están a la base del peligro para su bienestar ${ }^{6}$.

Respondiendo a esta lógica, el modelo americano de protección de la infancia impulsa la instalación de dispositivos técnicos altamente jerarquizados que se despliegan en una "lógica del hacer" que posterga irreparablemente los espacios de reflexión y cuestionamiento de las bases. Ante la efectiva urgencia de la precaria situación en que se encuentra gran parte de los niños y niñas de la región, los proyectos de intervención se redactan de una forma bastante más expedita que con la frecuencia que se crean instancias de discusión o se fomentan los espacios de investigación ${ }^{7}$.

Este modelo de gestión regional va a caracterizarse por el alcance de un alto nivel de especialización y por la incorporación de nuevas disciplinas susceptibles de aportar al diseño de las sucesivas intervenciones para el campo de la familia y la infancia. Estos dispositivos van a estructurarse en una "relación de servicio" y serán orientados por la lógica de "proyectos". Las políticas impulsadas en esta línea promueven un tipo de intervención de duración limitada y con un alcance invariablemente menor a los potenciales beneficiarios.

Las intervenciones se desarrollan en la línea del acompañamiento y el seguimiento, las ayudas personalizadas o la creación de instancias de participación, entre otras formas que se alinean con una lógica de contrato en que las familias se comprometen a "devolver"

6 De ahí la clásica expresión que escuchamos en contextos académicos o políticos donde se discute la protección de la infancia: "los niños ricos no tienen derechos". No tienen justamente porque no los necesitan ni les incumben.

7 Sería interesante, por ejemplo, investigar la proporción en que se destina el presupuesto de los entes rectores en infancia en la región para el financiamiento de actividades técnicas versus las de investigación. 
algo en retribución de los beneficios otorgados. Este modelo perpetúa una desigualdad simbólica cada vez que las familias se descubren incapaces de responder a las retribuciones exigidas, incapacidad que obviamente se desprende de la condición precaria que los llevó a ser "beneficiarias" de la ayuda social en primera instancia. Finalmente, si las familias son definidas como una entidad libre y autónoma, es para que las políticas públicas puedan exigir de ellas responsabilidad y una "actitud activa" frente a su condición precarizada.

En efecto, ahora que los derechos ya no están asegurados, se requiere de una "activación" para obtenerlos: encuadrarse sin objeción a la lógica externa que estipula de antemano "el interés superior del niño", exponer una y otra vez la vida privada para justificar con una "miseria sostenida" las ayudas otorgadas, especializarse en los sistemas burocráticos de asignación de recursos para cumplir con todos los trámites solicitados, explotar activamente una relación de competencia con los pares para estar primeros en la lista de beneficios, entre otros esfuerzos individualistas que fecunda esta gestión. Finalmente, este abanico de formas que encarna el fomento de esta "actitud activa" responde siempre a una obligación pero en ningún caso aumenta los espacios de "autonomía" que se habían planteado como el objetivo de base para la intervención. Por lo demás, la imposibilidad de visualizar una estabilidad para las familias (porque ni aún desplegando al máximo su "activación" ellas pueden tener certeza de ser capaces de cumplir "adecuadamente" su función o que los recursos seguirán disponibles) contribuye en gran medida a la repetición de esta dependencia.

Finalmente, toda la vida cotidiana de las familias se racionaliza, creándose servicios específicos que burocratizan una parte cada vez mayor de su experiencia. En esa línea, las estructuras preocupadas por la protección de los derechos de niños, niñas y adolescentes se fragmentan y la infancia, en fin, también se burocratiza, pasando a ser una variable desintegrada en la serie discontinua de objetos susceptibles de intervención.

Burocratizar la infancia permite, finalmente, segmentar a niños, niñas y adolescentes, aislándolos de su relación social y de su intrínseca pertenencia a la sociedad, con el objetivo de obviar una interrogante mayor, justamente aquella pregunta inevitable por el modelo que subyace a su condición de "infancia en peligro" o "infancia peligrosa": "Digámoslo sin eufemismos: analizar el hecho social del ser pobre o, más particularmente, de la infancia pobre, sin relacionarlo con los procesos económicos de concentración de ingresos, riqueza y poder, es como trabajar por y para su reproducción" (Bustelo, 2011: 33).

En este sentido, aunque se desarrollen intervenciones en nombre del "niño en riesgo", lo que no deja de llamar la atención es la radicalidad con que se evade el problema de la redistribución de los ingresos y la riqueza, que es finalmente la base de una explicación para la infancia como la conocemos: "(...) se plantea ingenuamente que lo que les sobra a unos es exactamente lo que necesitan otros y que, por lo tanto, sería sólo suficiente poner en contacto al donante y al necesitado" (Bustelo, 2011: 39).

En efecto, bien sabemos que la política social no debe buscar repartir armónicamente el todo entre las partes, porque esa repartición no existe: "la política no es, entonces, el orden arbitral que restaura una armonía de manera proporcional sino que, en las antípodas, 
intenta alterar el orden supuestamente armónico que deja afuera a los que no tienen parte" (Bustelo, 2011: 33).

La certeza que tenemos hasta aquí es que soslayar este nivel de lectura para las políticas sociales o programas sectoriales dirigidos a la infancia en América es un gesto plenamente coherente con el modelo asistencialista y compensatorio que, en el caso de los Estados periféricos, son lógicas constitutivas del espiral de empobrecimiento. En sociedades empobrecidas como Latinoamérica, las políticas del Estado asistencialista encubren las reales causas de los problemas sociales y postergan sus soluciones debido, fundamentalmente, a factores asociados a la estructura y composición del Estado, tradicionalmente construidos a espaldas de la sociedad (Unda Lara, 2003).

Se trataría entonces de una interdependencia asimétrica en la cual la dimensión económica deja de ser regulada por la política social y pública, para dar paso a un proceso inverso en el que la política económica organiza el alcance y las vías de la política social.

En materia de Derechos Humanos en el continente americano, esto sigue develando un contexto de especialización con arreglo a fines de reproducción social, donde muchos de sus campos particulares, como la infancia, han sido tratados como meros objetos de intervención más que como objetos teóricos y móviles. En este sentido, estamos frente a una ampliación e intensificación de la presencia y utilización de técnicas sociales diseñadas para "subsanar" el problema de la infancia y que se distancian cada vez más de reflexionar sobre sus enclaves contextuales y democráticos.

\section{BIBLIOGRAFÍA}

Bustelo, Eduardo (2011): El recreo de la infancia. Argumentos para otro comienzo, Siglo XXI, Buenos Aires.

Carreras, Sandra y Bárbara Potthast (coord.) (2005): Entre la familia, la sociedad y el Estado: niños y jóvenes en América Latina (sig/o XIX-XX), Biblioteca Iberoamericana, Madrid.

Donzelot, Jacques (1977): La Police des Familles, Éditions de Minuit, Paris.

Fass, Paula (2011): "A Historical Context for the United Nations Convention on the Rights of the Child", The Annals of the American Academy of Political and Social Science, 633 (1), pp. 17-29.

Guy, Donna (1998): “The Pan American Child Congresses, 1916 to 1942: Pan Americanism, Child Reform, and the Welfare State in Latin America", Journal of Family History, 23 (3), pp. 272-291. (1998a): "The Politics of Pan-American Cooperation: Maternalist Feminism and de Child Rights Movement, 1913-1960", Gender \& History, 10 (3), pp. 449-469.

IIAPI (1929, enero): "Boletín", Tomo II, №3, enero, Montevideo.

(1948, marzo): "Boletín", Tomo XXII, № 1, marzo, Montevideo.

(1949): "Acta de la reunión anual del Consejo Directivo", Montevideo.

(1956, diciembre): "Boletín", Tomo XXX, № 4, diciembre, Montevideo.

(1959): "Acta de la reunión anual del Consejo Directivo", Bogotá. 
(1979, marzo-diciembre): “Boletín”, Tomo LIV, № 208/2011, marzo-diciembre, Montevideo. (1990, julio): "Boletín", Tomo LXIII, № 230, julio, Montevideo.

(2000, febrero): “Informe de Labores, 1996-2000", presentado por el Director General Rodrigo Quintana, Montevideo.

Llobet, Valeria (2010): ¿Fábrica de niños? Las instituciones en la era de los derechos de infancia, Noveduc, Buenos Aires.

Manarelli, María Ema y Pablo Rodríguez (coord.) (2007): Historia de la infancia en América Latina, Universidad de Externado Colombia.

Opertti, Didier (2009): “Conmemoración del 82 Aniversario de la fundación del IIN. Los 60 años de su integración como Organismo Especializado de la OEA", en http://www.xxcongresopanamericano. org/presentacion/espanol/ [Consultado el 15/09/2011].

Pupavac, Vanessa (2001): "Misanthropy Without Borders: The International Children's Rights Regime", Journal of Disasters Studies, Policy and Management, 5 (2), pp. 95-112.

Unda Lara, René (2003): "Sociología de la infancia y política social: ¿compatibilidades posibles?", en http://foros.uexternado.edu.co/portal/html/modules.php?name=News\&file [Consultado el 10/11/11].

Recibido: 26-09-2012

Aceptado: 29-06-2012 